\title{
Structure and magnetic resonance parameters of the cation-radicals of methionine as studied by the CIDNP magnetic field dependence
}

\author{
Sergey E. Korchak, ${ }^{\text {abc }}$ Konstantin L. Ivanov, ${ }^{\text {ab }}$ Alexandra V. Yurkovskaya, ${ }^{a}$ and Hans- \\ Martin Vieth ${ }^{\mathrm{c}^{*}}$ \\ a International Tomography Center, Novosibirsk 630090, Russia \\ ${ }^{b}$ Novosibirsk State University, Novosibirsk 630090, Russia \\ ${ }^{c}$ Institut für Experimentalphysik, Freie Universität Berlin, \\ Arnimallee 14, D-14195 Berlin, Germany \\ E-mail: hans-martin.vieth@physik.fu-berlin.de
}

\section{Dedicated to Prof. P. T. Narasimhan on the occasion of his $75^{\text {th }}$ birthday}

(received 27 Jun 04; accepted 29 Dec 04; published on the web 29 Jan 05)

\begin{abstract}
The cation radicals formed when methionine and $\mathrm{N}$-acetylmethionine quench the photo-excited triplet state of 4-carboxybenzophenone in aqueous solution are characterized by their g-factors and hyperfine coupling constants. It is shown that the radicals exist as either cyclic or linear structures depending on the amino acid and the $\mathrm{pH}$ of the medium. The study is based on the analysis of the dynamic nuclear polarization generated in the spin-selective decay of the transient radicals and its dependence on the external magnetic field. The requirements and scope of this method are discussed.
\end{abstract}

Keywords: Methionine, $N$-acetylmethionine, 4-carboxybenzophenone, CIDNP, electron transfer

\section{Introduction}

The NMR spectra of reaction products resulting from a radical reaction often exhibit an anomalous intensity pattern because of transient spin polarization generated in the course of the reaction ${ }^{1}$. While other well-known mechanisms of dynamic nuclear polarization, e.g. the Overhauser $^{2,3}$ effect, rely on pumping of electron spin transitions this Chemically Induced Dynamic Nuclear Polarization (CIDNP) results from spin-selective reaction steps and it has been widely used for analyzing intermediate reaction stages. A particular application is the probing of protein structure ${ }^{4,5}$ and folding. ${ }^{6-8}$ For such protein studies, CIDNP is created by reactions involving reversible electron or hydrogen atom transfer between a photo-excited dye molecule and CIDNP-active amino acid residues. The polarization of the CIDNP-active residues in the 
protein depends on their accessibility for the probing dye molecule. Therefore, by analyzing the protein CIDNP one obtains the accessibilities of residues on the protein surface. For some time it was believed that among the 20 common amino acids only three (histidine, tyrosine, and tryptophan) are sufficiently CIDNP-active, that is, acquire substantial CIDNP when exposed to excited dye molecules. However, more recent experiments performed on methionine (Met) with flavin mononucleotide used as a dye revealed that methionine is also CIDNP-active. ${ }^{9,10}$ The intensity of methionine CIDNP, however, is increased when 4-carboxybenzophenone (CBP) is used as the dye. ${ }^{11,12}$

While the CIDNP kinetics and field dependencies of histidine, tyrosine and tryptophan have been studied in detail, there is still insufficient knowledge concerning the CIDNP of Met, the pathways and the kinetics of the reaction of Met with CBP, and the magnetic resonance properties of the involved radical species of Met. In this context the present work is aimed at studies of the photochemical reactions of Met and N-acetylmethionine (NMet) with CBP by analyzing the dependence of the observed CIDNP on the external magnetic field. Usually, the CIDNP is studied at the fixed external magnetic field of the NMR spectrometer (typically several Tesla). Such a practice limits the potential of the CIDNP method because the polarization efficiency strongly depends on the external magnetic field strength. By contrast, we have performed our experiments at variable magnetic field. This opens the opportunity to optimizing the field strength to enhance the signals of particular amino acid residues in the CIDNP studies of proteins and to extracting magnetic resonance parameters of the radical intermediates responsible for the CIDNP formation. While the high field part of the CIDNP depends on both the g-factor and the hyperfine interactions (HFI) of the radicals, the CIDNP at low field is conditioned solely by the HFI. Therefore, the analysis of the low field CIDNP provides information on the HFI constants. Once the HFI constants have been determined, the g-factor of the radical can be obtained from the CIDNP at high field. Thus, such a strategy allows one to extract the desired magnetic resonance parameters from the field dependence of the CIDNP. Because all individual field dependencies of the protons in a radical pair are simulated together with one common set of parameters, the fitting procedure is sensitive in spite of the large number of parameters.

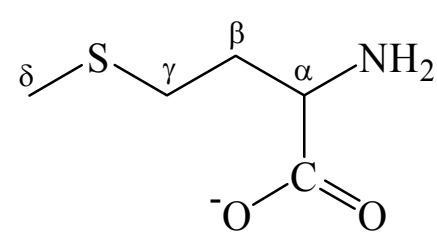

Methionine (Met)

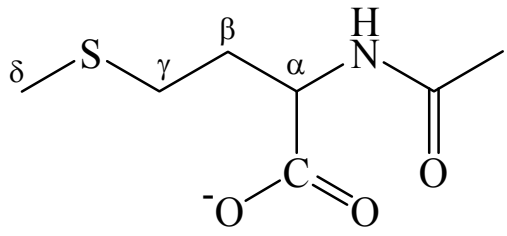

$\mathrm{N}$-acetylmethionine (NMet)

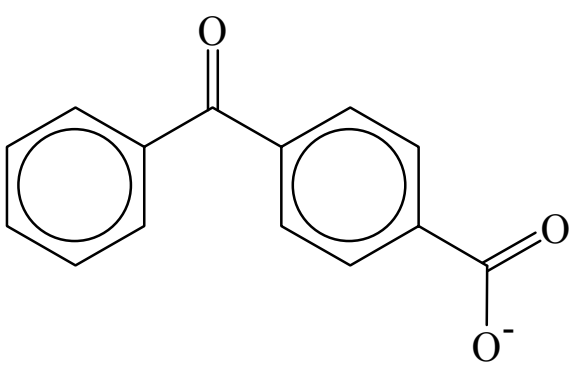

4-carboxybenzophenone (CBP)

Chart 1. Structures of the compounds under study. 
The structures of Met, NMet and CBP are shown in Chart 1. The carboxyl group of 4carboxybenzophenone has a $K_{\mathrm{a}}$ of 4.2 , hence, in the $\mathrm{pH}$ range used in our research $(\mathrm{pH} 6 \ldots 12)$ CBP exists in its deprotonated anionic form. Met has a $K_{a}$ of 9.27 (in $\mathrm{H}_{2} \mathrm{O}$ ) for its amino group, which therefore exists either in its protonated form as $\mathrm{NH}_{3}{ }^{+}$at a $\mathrm{pH}$ below this value or otherwise as $\mathrm{NH}_{2}$ with a lone electron pair above this value. The nitrogen of NMet is not protonated in this $\mathrm{pH}$ range and retains the structure shown in Chart 1 throughout.

\section{Results and Discussion}

\section{a) CIDNP Spectra}

After photo excitation of CBP and intersystem crossing the excited triplet state of CBP is quenched by the amino acid via electron transfer, presumably from the sulfur atom of the amino acid, to the oxygen atom of ${ }^{\mathrm{T}} \mathrm{CBP}$. The result of this reaction is the formation of a spin correlated radical pair consisting of an amino acid cation radical and the radical anion of CBP. The quenching rate constant has been measured as $\mathrm{k}_{\mathrm{q}}=2.6 \times 10^{9} \mathrm{M}^{-1} \mathrm{~s}^{-1} \cdot{ }^{13,14}$ Back electron transfer from the anion radical of $\mathrm{CBP}$ to the cation radical of the amino acid leads to nuclear spin polarization of both, amino acid and CBP, due to the hyperfine interaction with the unpaired electrons.

According to the assumed spin density distribution around the $\mathrm{S}$ atom of the Met and NMet radicals (see Chart 2), only the $\delta$ and $\gamma$ protons, which are located close to the radical center, are expected to be polarized, whilst for CBP all of the ring protons can show CIDNP due to delocalization of spin density in the aromatic system. The NMR signals of the nuclei that exhibit polarization generated in the course of the reversible photoreaction have the same chemical shifts as in the non-polarized starting material and thus can be easily differentiated from polarization formed in competing reactions.
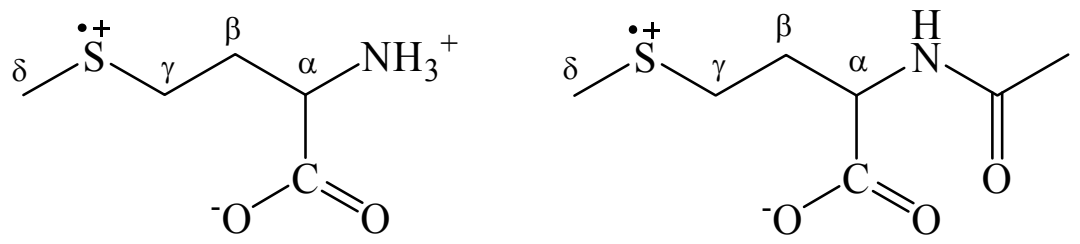

Chart 2. The structures of S-centered linear Met and NMet cation radicals.

Figure 1 shows the thermally polarized NMR spectra and the CIDNP spectra of the Met/CBP system at $\mathrm{pHs} 11.5$ and 6.5. The CIDNP spectra represent the difference between NMR spectra recorded with and without light irradiation. In neutral $(\mathrm{pH} \mathrm{6.5)} \mathrm{solution} \mathrm{in} \mathrm{the} \mathrm{case} \mathrm{of} \mathrm{Met} \mathrm{and} \mathrm{in}$ both neutral and basic ( $\mathrm{pH}$ 11.5) solutions in the case of NMet, the expected behavior is seen. Across the full magnetic field range, polarization of neither the $\alpha$ protons nor the $\beta$ protons was not observed, but the polarization of both the $\gamma$ and $\delta$ protons was maintained. This is an 
indication that indeed the spin density is located mainly on the sulfur atom, whereas the $\alpha$ and $\beta$ protons have negligibly small hyperfine constants. From these results, one can conclude that for Met in neutral solution and for NMet in neutral and basic solutions the S-centered cation radicals shown in Chart 2 are responsible for CIDNP formation.

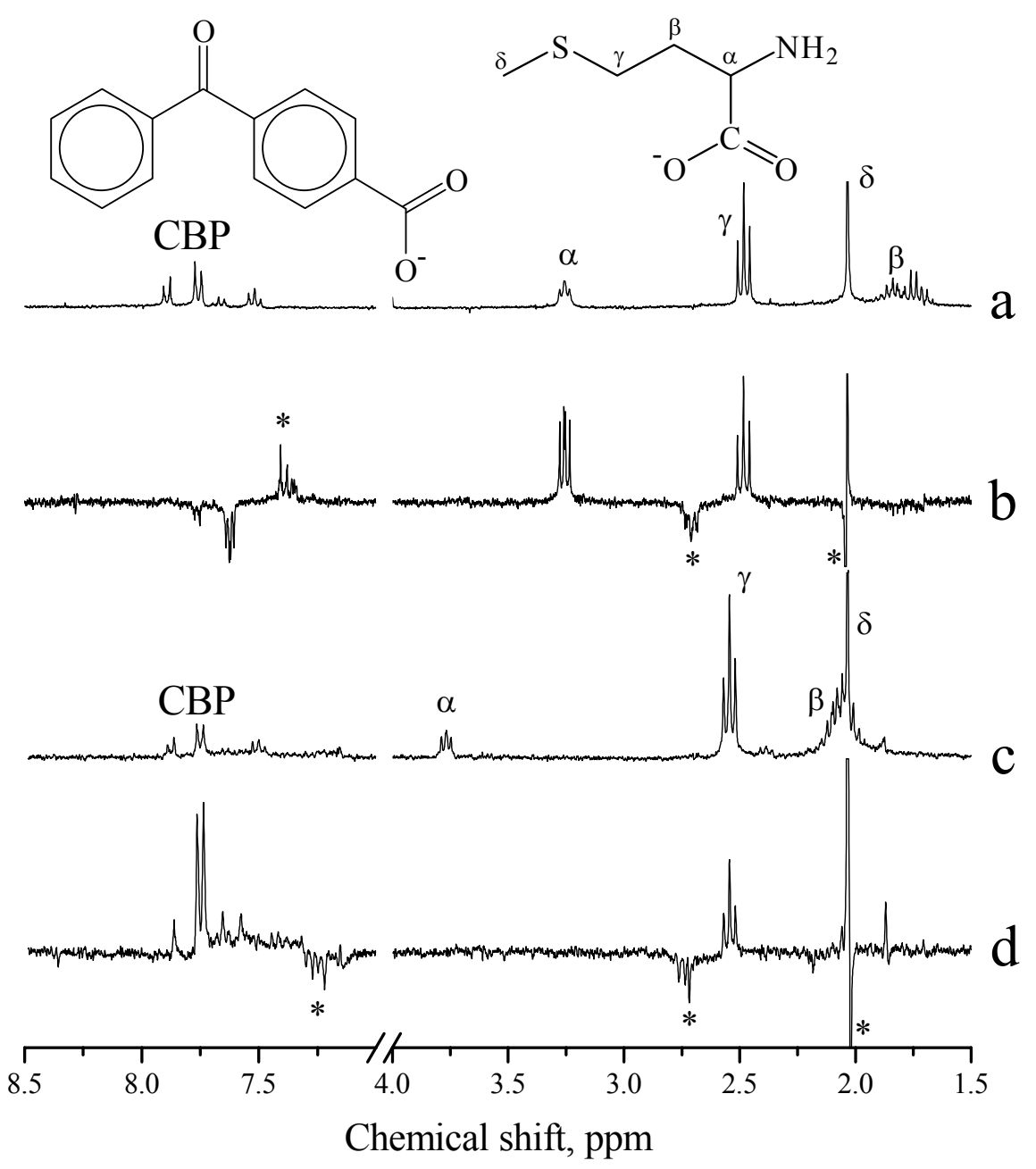

Figure 1. ${ }^{1} \mathrm{H}$ NMR spectra (Boltzmann polarization) of the Met/CBP system at $\mathrm{pH} 11.5$ (a) and 6.5 (c), and CIDNP spectra at $\mathrm{B}_{\mathrm{pol}} 2.4 \mathrm{~T}$ and $\mathrm{pH} 11.5$ (b) and at $\mathrm{B}_{\mathrm{pol}} 0.7 \mathrm{~T}$ and $\mathrm{pH} 6.5$ (d). Asterisks indicate signals of side products.

For Met in basic solution, the situation is distinctly different. Here, across the entire magnetic field range polarization was detected not only for the $\delta$ and $\gamma$ protons of Met but also for the $\alpha$ proton. This observation is in full accordance with that by Goez et al. ${ }^{11,12}$ Such a polarization is feasible only for a radical with sufficient spin density at the $\alpha$-position. A likely candidate for such a species is the cation radical of Met with a five-membered ring and a three-electron bond 
between $\mathrm{S}$ and $\mathrm{N}$ atoms as shown in Chart 3 (a) and originally suggested by Hiller et al. ${ }^{15}$ to explain the results obtained from pulse radiolysis experiments. Polarization of the $\alpha$ proton in the absence of $\beta$-polarization is an indication for the existence of the cyclic structure of the involved radical of Met. The formation of this cyclic structure arises from intramolecular reaction of a positively charged radical center at the sulfur atom with the partly negatively charged nitrogen atom which has a lone electron pair at this $\mathrm{pH}$.

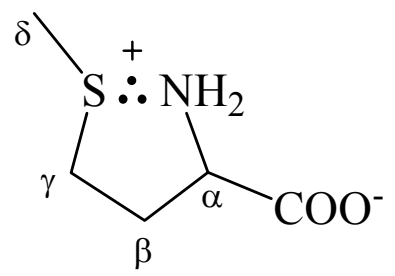

a

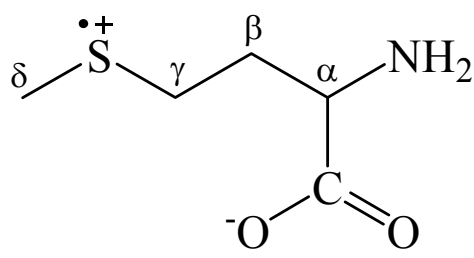

b

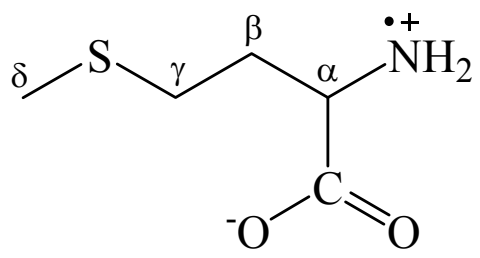

c

Chart 3. Cyclic and linear structures of the Met cation radical at basic conditions.

However, the same polarization pattern could, in principle, result, if two parallel reaction pathways with two different radical pairs were involved in the triplet quenching process. In this case the first reaction occurs with electron transfer from the sulfur atom of the amino acid with the formation of an S-centered radical, whereas the second one proceeds from the lone pair on the nitrogen with the formation of an aminyl radical, ${ }^{16}$ as it is shown on Chart 3 by the structures (b) and (c), respectively. As evidence for the formation of the cyclic radical structure the ratio of polarization level of the protons in the $\alpha$ and $\gamma$-position of methionine and its variation with the $\mathrm{pH}$ were taken by Goez and Rozwadowski, ${ }^{12}$ but a similar $\mathrm{pH}$ dependence is expected also for the relative contribution to polarization by the corresponding protons of the two linear radicals. Moreover, our recent time-resolved CIDNP measurements on the kinetics of this reaction at different $\mathrm{pH}$ show that the assumption ${ }^{12}$ of CIDNP creation exclusively at the geminate stage is unsustainable and that the $\mathrm{pH}$ dependence of the stationary CIDNP signal is not at all given by the interconversion of different radicals of methionine at the geminate stage.

\section{b) CIDNP field dependence}

A possible way to discriminate the occurrence of one cyclic or two linear intermediates is to determine the g-factor of the radicals so formed. The most direct way for measuring the g-factor is EPR, but because of their high reactivity and, consequently, low concentration the cation radicals of methionine have not yet been detected in aqueous solution. An alternative way to obtain the desired information on the g-factors of the participating radicals and on the reaction pathway is to utilize the dependence of CIDNP on the external magnetic field. Standard CIDNP theory relates the maxima of polarization at high magnetic field $\mathrm{B}_{0}$ to the difference in $\mathrm{g}$-factor $\Delta \mathrm{g}=\left(\mathrm{g}_{\mathrm{m}}-\mathrm{g}_{\mathrm{d}}\right)$, where $\mathrm{g}_{\mathrm{m}}$ and $\mathrm{g}_{\mathrm{d}}$ stand for the $\mathrm{g}$-factor of the methionine radical and of the dye radical, respectively. Since the g-factor of the S-centered radical of structure (b) is expected to be 
considerably larger than that of the aminyl radical of structure (c), the maxima of CIDNP of these radicals are expected to appear at very different $\mathrm{B}_{0}$ so that they can be resolved in the field dependencies of the methionine protons. Alternatively, if only the cyclic radical is involved, one common field of maximum polarization for all protons of methionine is expected with its location determined by the g-factors of the cyclic cation radical and the radical of the dye.

From Figure 2, where the field dependencies of the $\alpha, \gamma$ and $\delta$ protons of methionine at $\mathrm{pH}$ 11.5 are shown in the range between $0.1 \mathrm{mT}$ and $7 \mathrm{~T}$ it is evident that only single polarization maxima appear. Therefore any significant contribution from S-centered or N-centered linear radicals can be excluded at basic $\mathrm{pH}$, the cyclic cation radical is the dominant reaction intermediate. In the case of Met in neutral solution the formation of the cyclic cation radical is impossible due to the lack of the lone electron pair at nitrogen atom because of the protonation of the amino group. In case of NMet in neutral and basic solution probably the lone pair of the nitrogen interacts with the $\pi$-orbital of the carbonyl group and thus cannot be used for the formation of a bond between the $\mathrm{S}$ and $\mathrm{N}$ atoms. This is the same reason why acetylated amino groups can not be protonated under usual conditions.

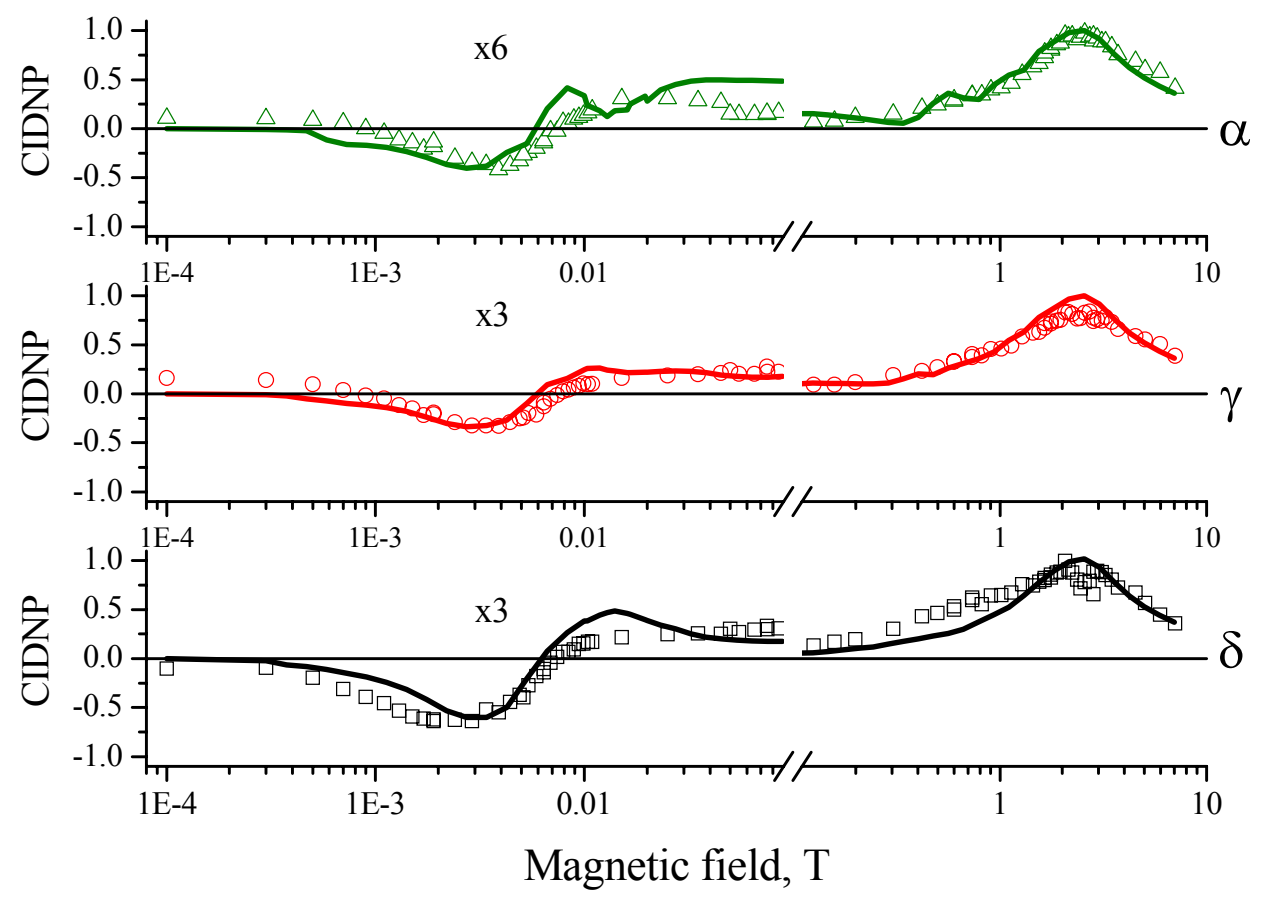

Figure 2. Field dependence of the $\alpha(\triangle), \gamma(\mathrm{O})$ and $\delta(\square)$ protons of methionine at $\mathrm{pH}$ 11.5. Lines correspond to the simulated CIDNP of the $\alpha$ proton (green), the $\gamma$ protons (red), and the $\delta$ protons (black) with the parameters for the cyclic Met cation radical shown in Table 1. Scaling of the amplitudes as described in the Experimental Section.

For quantitative evaluation we followed the approach outlined and determined the HFI constants and the g-factor of the cation radical from the CIDNP field dependence and the 
corresponding optimized simulation as shown in Figure 2 for methionine at $\mathrm{pH} 11.5$ and in Figure 3 for methionine and $\mathrm{N}$-acetylmethionine at $\mathrm{pH}$ 6.5. Since CIDNP depends on the difference $\Delta \mathrm{g}$ between the radical pair partners, the g-factor of one radical species can only be determined with reference to that of its partner. In our case there is the problem that the g-factor of the CBP radical is unavailable from the literature. However, Säuberlich et al. ${ }^{17,18}$ measured the g-factors of both benzophenone (BP) (2.0033) and of 3,3',4,4'-benzophenonetetracarboxylic acid (2.0035). We consider it reasonable to assume that the g-factors of the radicals BP and CBP do not differ much and take $\mathrm{g}_{\mathrm{CBP}}=2.0033$.

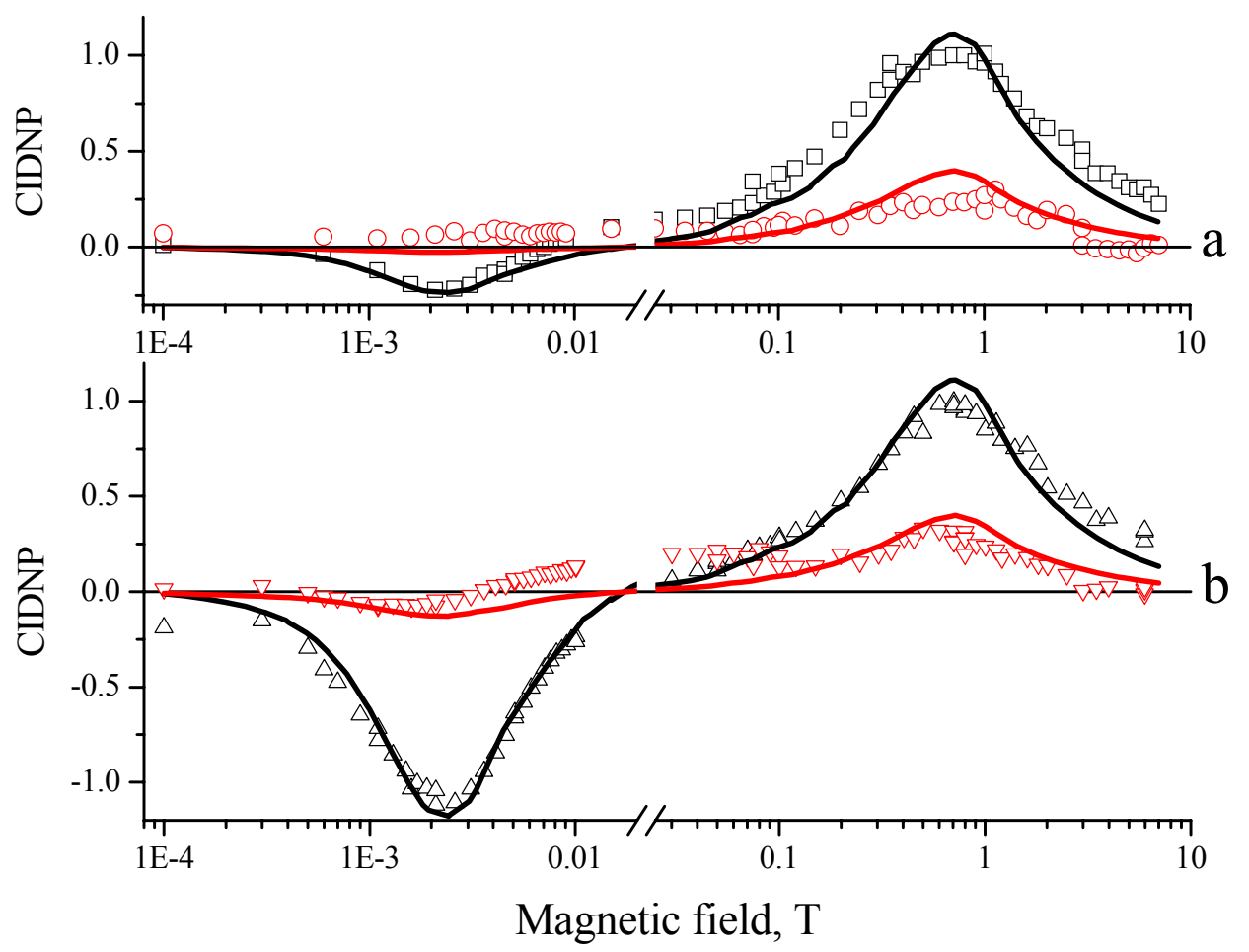

Figure 3. Field dependence at $\mathrm{pH} 6.5$ of a) $0 \delta(\square)$ and $\gamma(\mathrm{O})$ protons of N-acetylmethionine. b) $\delta$ $(\triangle)$ and $\gamma(\nabla)$ protons of methionine. Lines correspond to the simulated CIDNP of $\delta$ protons (black) and $\gamma$ protons (red) with parameters for linear Met and NMet cation radicals accordingly shown in Table 1. Scaling of the amplitudes as described in the Experimental Section.

As g-factor of the cyclic cation radical we obtain $\mathrm{g}=2.0064$, which is significantly smaller than the typical g-value of S-centered organic radicals (2.0100) indicating that part of the spin density is located at the nitrogen atom. The best fitting parameters for the Met CIDNP field dependence are listed in Table 1 . We assumed further that all three $\delta$ protons have equal HFI constants due the fast rotation of the methyl group.

The result that the HFI constants of the $\alpha, \gamma$ and $\delta$ protons are of comparable magnitude (also having the same sign) gives additional evidence for a bond between $\mathrm{S}$ and $\mathrm{N}$ in the cation radical. 
The large effective hyperfine constant $\mathrm{A}_{\text {eff }}=3.5 \mathrm{mT}$ of the residual nuclei is interpreted as due to the large HFI constant at the nitrogen position where much spin density is located.

At $\mathrm{pH}=6.5$, with the g-factor of the CBP radical taken as 2.0033 we obtain $\mathrm{g}=2.0100$ as the g-factor of the Met cation radical, which agrees with typical g-factors of S-centered organic radicals $(g=2.010)$. The HFI constants of the linear Met and NMet cation radicals are also included in Table 1.

In what regards the reaction with a photo-excited dye $\mathrm{N}$-acetylmethionine is close in its properties to those Met residues present in proteins; hence the results presented here can be used for optimizing the conditions in analogous experiments on proteins. As an example, the maximum of the CIDNP formation is expected to occur at $0.7 \mathrm{~T}$ in the high field range and at 2.6 $\mathrm{mT}$ in the low field range. Accordingly, by proper setting of the polarization field it is possible to selectively enhance methionine residues in the protein CIDNP spectra to optimize the detection sensitivity.

Table 1. g-Factors and HFI constants used for the simulation

\begin{tabular}{cccccc}
\hline Cation radical & g-factor & $\mathrm{A}_{\alpha,} \mathrm{mT}$ & $\mathrm{A}_{\gamma}, \mathrm{mT}$ & $\mathrm{A}_{\delta,} \mathrm{mT}$ & $\mathrm{A}_{\text {eff, }} \mathrm{mT}$ \\
\hline Cyclic Met & 2.0064 & 1.0 & 0.8 & 0.7 & 3.5 \\
Linear Met & 2.0100 & - & 1.0 & 0.7 & 2.0 \\
NMet & 2.0100 & - & 1.0 & 0.7 & 2.0 \\
\hline
\end{tabular}

\section{Conclusions}

The present work describes the results of a comprehensive study of the magnetic field dependence of the CIDNP formed from the reactions of Met and NMet with CBP in aqueous solution. Field dependencies of the CIDNP formed in the reaction of Met with triplet excited CBP have been measured in the region $0 \ldots 7$ Tesla in both neutral and basic solutions. An important factor is the variability of $B_{\text {pol }}$ over a wide range: the low field data are necessary for HFI analysis, and hence, in combination with measurements at high field, g-factors can be determined. Our results for neutral and basic solution give clear evidence that two structures of the Met cation radical are formed having different distributions of spin density. At a $\mathrm{pH}$ above 9 , the Met cation radical exists in a cyclic form with a two-center three-electron bond between the nitrogen and sulfur atoms, whilst in solutions below $\mathrm{pH} 9$ the Met cation radical has a linear structure. Comparison of the experimental results with model calculations of the CIDNP field dependencies allowed us to obtain the $g$-factors of the cyclic (2.0064) and the linear (2.0100) forms of the Met and NMet cation radical. Hyperfine constants of both forms of the Met cation radical were also determined. The high sensitivity of the CIDNP field dependence with respect to changes in $\Delta \mathrm{g}$ results in high accuracy of the parameters obtained and makes the analysis of CIDNP very suitable for characterizing short lived paramagnetic reaction intermediates. 


\section{Experimental Section}

General Procedures. A detailed description of the experimental setup has been provided elsewhere. ${ }^{19,20}$ The mechanical field cycling setup allows the detection of high resolution NMR spectra under permanent slow sample rotation $(0-150 \mathrm{~Hz})$ at $B_{0}=7 \mathrm{~T}$ and light irradiation for polarization at any desired magnetic field strength $B_{\text {pol }}$ between the earth magnetic field and $7 \mathrm{~T}$ by precise positioning of the sample in the stray field of the spectrometer cryomagnet. At a field below $0.1 \mathrm{~T}$, where a high homogeneity of the magnetic field across the sample volume is required, $B_{\mathrm{pol}}$ is set by control of the electric current through a pair of additional Helmholtz coils placed under the cryomagnet. A CIDNP experiment that employs mechanical field cycling contains three consecutive steps: (1) CIDNP generation by means of photoreactions at the desired field $B_{\text {pol }}$; (2) transfer of the polarized reaction products to the observation field $B_{0}$ of the NMR spectrometer (i.e. switching of the external magnetic field from $B_{\text {pol }}$ to $B_{0}$ ); (3) measurement of the FT-NMR at $B=B_{0}$. Since the field change occurs adiabatically, the populations of the individual nuclear spin eigenstates are conserved. During step (1) the sample is irradiated (typically for $0.5 \mathrm{~s}$ ) by a $\mathrm{XeCl}$ excimer laser at $308 \mathrm{~nm}$ with a repetition rate of $50 \mathrm{~Hz}$ and an energy of up to $100 \mathrm{~mJ} /$ pulse through a flexible liquid light guide with a $90^{\circ}$ prism on its top. The samples, containing either $0.02 \mathrm{M}$ Met or $0.005 \mathrm{M}$ NMet and $0.0025 \mathrm{M}$ of CBP (together with $0.02 \mathrm{M}$ of $\mathrm{Na}_{2} \mathrm{DPO}_{4}$ used as buffer) in $\mathrm{D}_{2} \mathrm{O}$, were prepared to yield an optical density of 2.0 at $308 \mathrm{~nm}$ for the 4-mm optical pathway inside the NMR sample tube. All samples were purged with pure nitrogen gas and sealed in standard $5 \mathrm{~mm}$ Pyrex NMR tubes. In order to prevent vortex formation and sample shaking during the transfer, a Teflon plug was inserted into the tube on top of the liquid. All chemicals were from Aldrich and used as received.

Computational details. Numerical simulation of the CIDNP field dependence allows quantitative determination of the magnetic resonance parameters of the radical species; the procedure employed has been described in detail elsewhere. ${ }^{21,22}$ The procedure is based on solving the stochastic Liouville equation of the radical pair by means of the Green function technique $^{23}$ and on utilizing the so-called low-viscosity approximation ${ }^{24}$ which implies the degree of singlet-triplet conversion to be relatively low in each nuclear sub-ensemble: $v \tau_{\mathrm{d}}<<1$ (here $v$ is the characteristic rate of S-T mixing and $\tau_{\mathrm{d}}$ is the time of diffusional displacement between the radical partners). Despite the low degree of S-T mixing during the radical pair lifetime the CIDNP is still higher than the thermal polarization by several orders of magnitude. In our simulations we have taken into account all protons ( $\alpha, \gamma$ and $\delta$ protons) of Met in a consistent quantum-mechanical manner, while the remaining hyperfine interaction with other magnetic nuclei of Met has been considered using a semiclassical approximation ${ }^{25}$ with the effective HFI constant $\mathrm{A}_{\text {eff }}$ in accordance with earlier work. ${ }^{21,22,26}$ The HFI constants of CBP are so small ${ }^{17,18}$ that their influence on the field dependence is negligible, hence they have not been taken into account. As a side note we want to mention that the simulations model only the geminate reaction stage, though secondary reactions in the bulk also contribute to the observed 
CIDNP. The main effect is the reduction of polarization at high field with respect to that at low field. Accordingly we changed the amplitude scaling factor at about $B_{\text {pol }}=0.1 \mathrm{~T}$ so that the calculated curves fit the experimental data at both the polarization maximum at high field and the minimum at low field. For $\mathrm{N}$-acetylmethionine at $\mathrm{pH} 6.5$ because of low signal intensity the scaling in the case of the $\gamma$ protons was related to that of the $\delta$ protons according to the corresponding curves of methionine.

\section{Acknowledgements}

The financial support by RFBF (project no. 02-03-32765), INTAS (project no. 02-2126) and the Russian Ministry of High Education (grant no. 2298.2003.3) is gratefully acknowledged. A.V.Y. is indebted to the Russian Science Support Foundation, S.E.K. thanks DAAD for financial support, K.L.I. is indebted to INTAS for financial support (grant no. YSF 2001/2-103) and the generous attitude toward him.

\section{References}

1. Salikhov, K. M.; Molin, Y. N.; Sagdeev, R. Z.; Buchachenko, A. L. Spin Polarization and Magnetic Effects in Chemical Reactions; Elsevier: Amsterdam, 1984.

2. Overhauser, A. W. Phys. Rev. 1953, 92, 411.

3. Chandrakumar, N.; Narasimhan, P. T. Mol. Phys. 1982, 45, 179.

4. Hore, P. J.; Broadhurst, R. W. Prog. Nucl. Mag. Res. Sp. 1993, 25, 345.

5. Kaptein, R.; Dijkstra, K.; Nicolay, K. Nature 1978, 274, 293.

6. Wirmer, J.; Kuehn, T.; Schwalbe, H. Angew. Chem. 2001, 113, 4378.

7. Hore, P. J.; Winder, S. L.; Roberts, C. H.; Dobson, C. M. J. Am. Chem. Soc. 1997, 119, 5049.

8. Dobson, C. M.; Hore, P. J. Nature Struct. Biol., NMR suppl. 1998, 5, 504.

9. Stob, S.; Kaptein, R. Photochem. Photobiol. 1989, 49, 565.

10. Lyon, C. E.; Lopez, J. J.; Cho, B.-M.; Hore, P. J. Mol. Phys. 2002, 100, 1261.

11. Goez, M.; Rozwadowski, J.; Marciniak, B. Angew. Chem., Int. Ed. 1998, 37, 628.

12. Goez, M.; Rozwadowski, J. J. Phys. Chem. A 1998, 102, 7945.

13. Bobrowski, K.; Marciniak, B.; Hug, G. L. J. Am. Chem. Soc. 1992, 114, 10279.

14. Hiller, K. O.; Mosloch, B.; Gobl, M.; Asmus, K.-D. J. Am. Chem. Soc. 1981, 103, 2734.

15. Bobrowski, K.; Hug, G. L.; Marciniak, B.; Kozubek, H. J. Phys. Chem. 1994, 98, 537.

16. Hug, G. L.; Bonifačić, M.; Asmus, K.-D.; Armstrong, D. A. J. Phys. Chem. 2000, 104, 6674.

17. Säuberlich, J.; Beckert, D. J. Phys. Chem. 1995, 99, 12520.

18. Säuberlich, J.; Brede, O.; Beckert, D. J. Phys. Chem. 1996, 100, 18101. 
19. Grosse, S.; Yurkovskaya, A. V.; Lopez, J.; Vieth, H.-M. J. Phys. Chem. A 2001, 105, 6311.

20. Grosse, S. Ph. D. Thesis, Free University of Berlin, 2000.

21. Ivanov, K. L.; Lukzen, N. N.; Vieth, H.-M.; Grosse, S.; Yurkovskaya, A. V.; Sagdeev, R. Z. Molecular Physics 2002, 100, 1197.

22. Ivanov, K. L.; Vieth, H.-M.; Miesel, K.; Yurkovskaya, A. V.; Sagdeev, R. Phys.Chem. Chem. Phys. 2003, 5, 3470.

23. Doktorov, A. B.; Purtov, P. A. Sov. Chem. Phys. 1987, 6, 484.

24. Osintsev, A. M.; Purtov, P. A.; Salikhov, K. M. Chem. Phys. 1993, 174, 237.

25. Schulten, K.; Wolynes, P. G. J. Chem. Phys. 1978, 68, 3292.

26. Osintsev, A. M.; Purtov, P. A.; Salikhov, K. M. Sov. Chem. Phys. 1992, 11, 1192. 